\title{
Development of a rabies virus-based retrograde tracer with high trans-monosynaptic efficiency by reshuffling glycoprotein
}

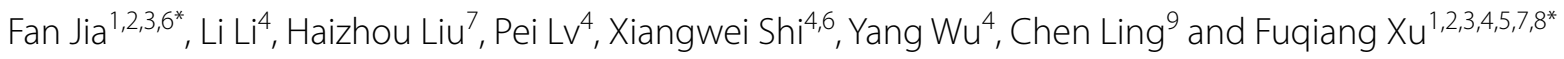

\begin{abstract}
Rabies virus (RV) is the most widely used vector for mapping neural circuits. Previous studies have shown that the RV glycoprotein can be a target to improve the retrograde transsynaptic tracing efficiency. However, the current versions still label only a small portion of all presynaptic neurons. Here, we reshuffled the oG sequence, a chimeric glycoprotein, with positive codon pair bias score (CPBS) based on bioinformatic analysis of mouse codon pair bias, generating $\triangle 0 G$, a further optimized glycoprotein. Our experimental data reveal that the ooG has a higher expression level than the oG in vivo, which significantly increases the tracing efficiency by up to 12.6 and 62.1 -fold compared to oG and B19G, respectively. The new tool can be used for labeling neural circuits Therefore, the approach reported here provides a convenient, efficient and universal strategy to improve protein expression for various application scenarios such as trans-synaptic tracing efficiency, cell engineering, and vaccine and oncolytic virus designs.
\end{abstract}

\section{Introduction}

Mapping neural circuits is a prerequisite to elucidate the mechanisms of brain functions. Several neurotropic viruses can infect neurons and spread across synapses between neurons, playing important roles in depicting the neurocircuit [1]. Among these virus-based tools, rabies virus (RV), encoding five proteins $(\mathrm{N}, \mathrm{P}, \mathrm{M}, \mathrm{G}$ and $\mathrm{L}$ ), is the most popular trans-monosynaptic viral tool to map the direct input networks of specific types of neurons in specific brain regions [2-6]. To realize cell type specific and trans-monosynaptic tracing, (i) the RV-G gene is deleted from genome (RV-delG); (ii) a chimeric EnvA gene is created by swapping the cytoplasmic part of $\mathrm{G}$ and wild type EnvA (a glycoprotein from ASLV-A;

\footnotetext{
*Correspondence: fan.jia@siat.ac.cn; fq.xu@siat.ac.cn

${ }^{1}$ Guangdong Provincial Key Laboratory of Brain Connectome and Behavior, CAS Key Laboratory of Brain Connectome

and Manipulation, The Brain Cognition and Brain Disease Institute (BCBDI), Translational Research Center for the Nervous System (TRCNS),

Shenzhen Institute of Advanced Technology, Chinese Academy of Sciences, Shenzhen 518055, China

Full list of author information is available at the end of the article
}

(iii) a cell line expressing the chimeric EnvA is prepared to package the genome of RV-delG to produce the pseudo-typed virus (EnvA-RV-delG); (iv) two adenoassociated virus (AAV) vectors (AAV-G, and AAV-TVAGFP), cre-dependently delivering G-gene, GFP, and TVA (a receptor of EnvA), are constructed separately; (v) EnvA-RV-delG can enter specific neurons infected by AAV-TVA-GFP; (vi) in the neurons co-infected by EnvARV-delG/AAV-G/AAV-TVA-GFP, the G protein transcomplements the package of RVG-RV-delG which is capable of trans-monosynaptically retrograde spreading to input neurons $[2,3]$. However, current $R V$ versions can only label a fraction of presynaptic neurons [7]. When AAV-G infects neurons, the G expression cassette cannot be replicated because of the AAV features (or the template number for G-gene is fixed), while EnvA-RV-delG enters the same neuron, the G-deleted RV genome can be reproduced and expressed according to the RV life cycle.

Therefore, a significant mismatch between the amounts of $\mathrm{G}$ protein and other RV proteins might exist, leading to few active RV particles and thus low tracing efficiency, at the same time, generating 
excessive unused viral proteins and thus high cytotoxicity. Conventional methods for improving the protein expression level are to use stronger promoters, optimize the gene sequence, or provide more copies of the gene [8-13]. Indeed, previous reports have provided evidence that elevating the expression level of the $G$ protein improves the tracing efficiency $[14,15]$. The protein synthesis machinery has a preference for how to better translate proteins in different species. Codon pair bias $(\mathrm{CPB})$ is a common phenomenon in various species for adjacent codon pairs, which appears with different frequencies in different species [16]. The favorite or unfavorite of a codon pair can be defined by its codon pair bias score (CPBS), defined as the ratio of observed frequency to the expected frequency. Every codon pair has a CPBS. A positive CPBS means that a codon pair is overrepresented, which may be preferred by the organism, whereas a negative CPBS may be unfavorable. Therefore, recoding a gene with positive CPBS may adjust protein expression. Many viruses have been attenuated by using the negative CPBS which results in decreased protein production [17-19], while only limited number of studies explored the effect of positive CPBS on viral properties. RV as a retrograde transmonosynaptic tracer is most commonly used to label neural circuits in mice, however, mice are not the reservoir host. This cross-species shift may have a profound impact on the expression of proteins encoded by RV. The amount of $\mathrm{G}$ is a limiting factor for trans-synaptic efficiency of RV. Here, we propose that better conditions to increase the G protein level could be achieved by using positive CPBS in mice. Indeed, this convenient method can elevate the $G$ expression level and improve RV trans-monosynaptic efficiency.

\section{Results}

The relationship of canine, mouse and human in CPB

$\mathrm{RV}$ has wide infection range, such as canine, mouse and human. Among these animals, canine is one of the RV reservoir hosts, which providing the opportunity that $\mathrm{RV}$ has adopted the CPB of canine during the long-term co-evolution. While, RV-based tools are usually used in mouse model in neuroscience research. Therefore, the codon pair score (CPS) of canine, mouse and human were calculated using previously reported method [17]. Briefly, a total of 45,094, 68,272 and 110,788 genes for canine, mouse and human were analyzed to calculate codon pair bias score for each of the 3721 codon pair $(61 \times 61$ codons). The CPS values of these three species are very similar (Fig. 1a-c), however, the relationships of the three species are different. The correlation coefficient value of codon pair preference between human and canine (Spearman rho $=0.9859)$ is closer than that between mouse and canine (Spearman rho $=0.9459)$, and also closer than that between human and mouse (Spearman rho $=0.9588)$. Next, we calculated the CPBS values for each gene of mouse and canine respectively and plotted the CPBS value against its gene length. We found that the majority of genes in mouse and canine have positive CPBS, averaged at 0.0651 and 0.0704 , respectively, which are similar to the human 0.0703 (Additional file 1: Fig. S1). Therefore, these data provide a basic for oG reshuffling (Fig. 2a).

\section{ooG has higher expression level than oG}

The oG, the best version of $G$ in current RV-based tools [15], was selected as a model for codon pair optimization based on codons pairs preference data from the mouse genome to improve its expression level. Based
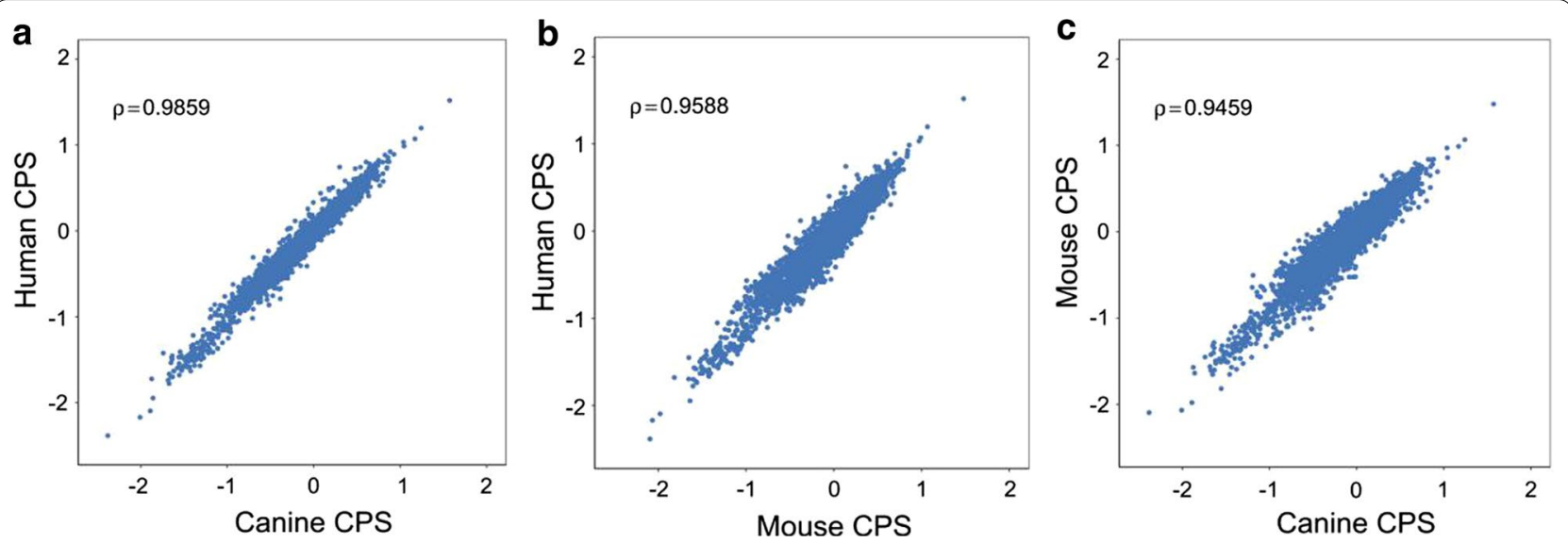

Fig. 1 Codon pair bias in three speices (human, canine and mouse). a-c Each steel blue dot represents one of the 3721 possible codon pairs and shows codon pair score (CPS) in the human, the canine and the mouse. The CPSs of these species were separately calculated using the available genes of the human $(110,788)(\mathbf{a})$, the canine $(45,094)(\mathbf{b})$, and the mouse $(68,272)(\mathbf{c})$ using a previous described method $[17]$ 


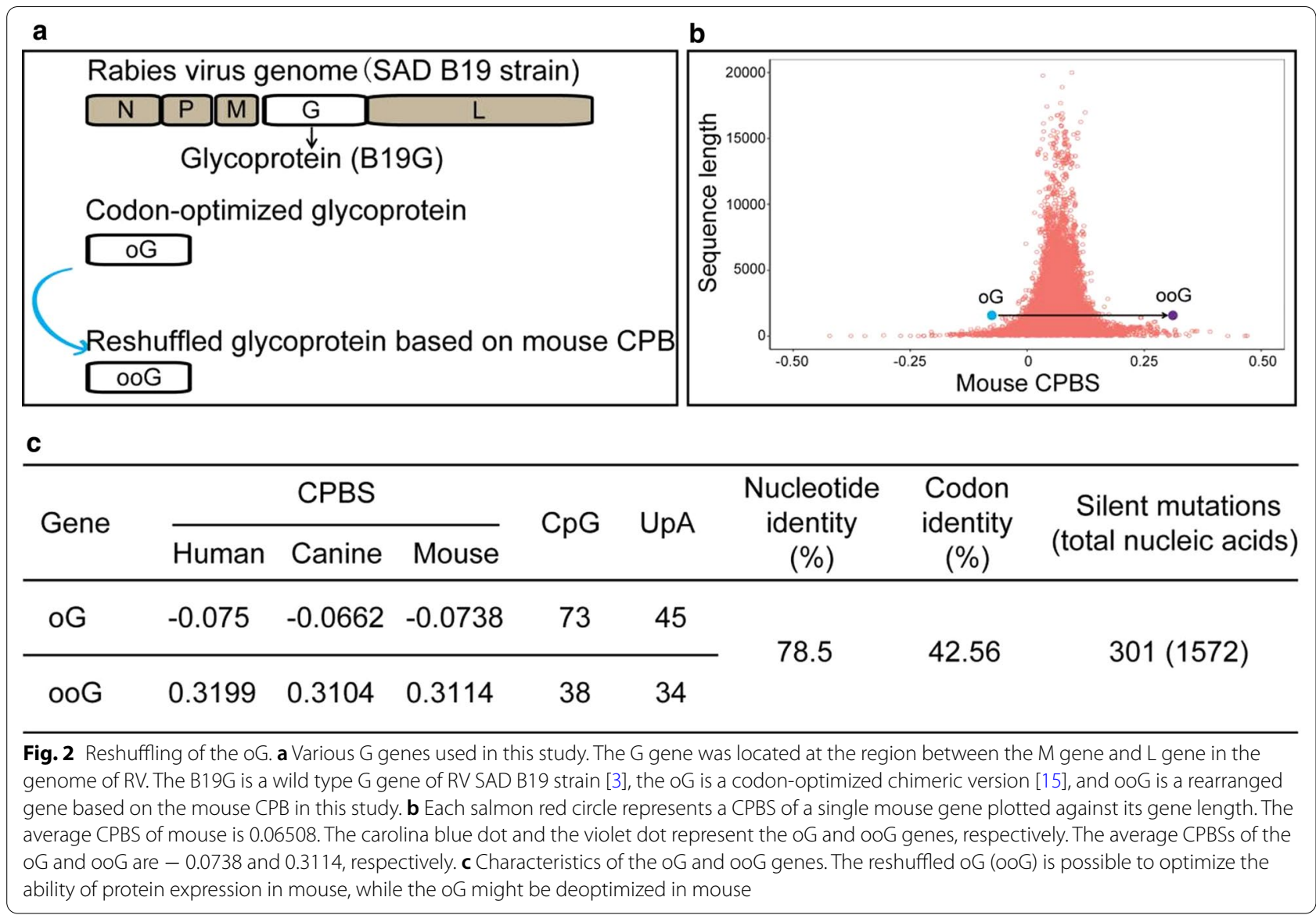

on the CPS obtained from the mouse, we attempted to reshuffle the oG sequence to improve oG's expression level, and named the new oG sequence as ooG (Additional file 1: Fig. S2). The median CPBS of oG and ooG is $-0.0738,0.3114$, respectively (Fig. 2b, c). The number of $\mathrm{CpG}$ and UpA are 73 and 45 for oG, 38 and 34 for ooG (Fig. 2c).

Next, the genes of the ooG and oG were commercially synthesized and cloned into the self-complementary AAV (scAAV) vector, respectively [20], in which the ooG or oG was expressed by the syn promoter (Fig. 3a). $150 \mu \mathrm{l}$ of scAAV-hsynP-ooG-bGHpA and scAAV-hsynP-oG-bGHpAwere injected into the ventral hippocampus (vHPC) region of C57BL/6 mouse, respectively. After three weeks, the brain tissue was collected and analyzed (Fig. 3b). Firstly, protein level was determined using antibody against $\mathrm{G}$, which is specific to the ooG and the oG (Fig. 3c). We found that the amount of ooG protein is higher than oG's (Fig. 3d). Furthermore, we found that the ooG mRNA copies are higher than oG's (Fig. 3e). Collectively, the ooG as a novel gene has higher protein expression level than oG.
ooG can enhance the trans-synaptic efficiency

Based on the data, we provide a hypothesis that the ooG has a potential ability of increasing RV trans-synaptic efficiency. To directly compare the transsynaptic efficiency of the ooG, oG, and B19G, the long-distance input data in known circuit were collected and analyzed by calculating the convergence index. ssAAV-EF1 $\alpha$-DIO-EGFPF2A-TVA-WPRE-bGHpA was co-injected with one of the three AAVs: scAAV-hsynP-ooG-bGHpA, scAAVhsynP-oG-bGHpAand scAAV-hsynP-B19G-bGHpA into the Vhpc region of Thy-1 cre mouse, respectively. After three weeks, the EnvA-pseudorabies (Enva-RV844) were injected into the same region. The brains were collected and processed seven days later.

We observed the main long-distance input regions are the paraventricular thalamic nucleus (PVA) and the medial septal nucleus (MS), which are known and consistent with the previous reports (Fig. 4a) [21]. Then the convergence index was calculated to directly compare the retrograde trans-synaptic efficiency (14) of these three $G$ proteins by using the number of input neurons (mRuby3 positive) divided by the number of starter 

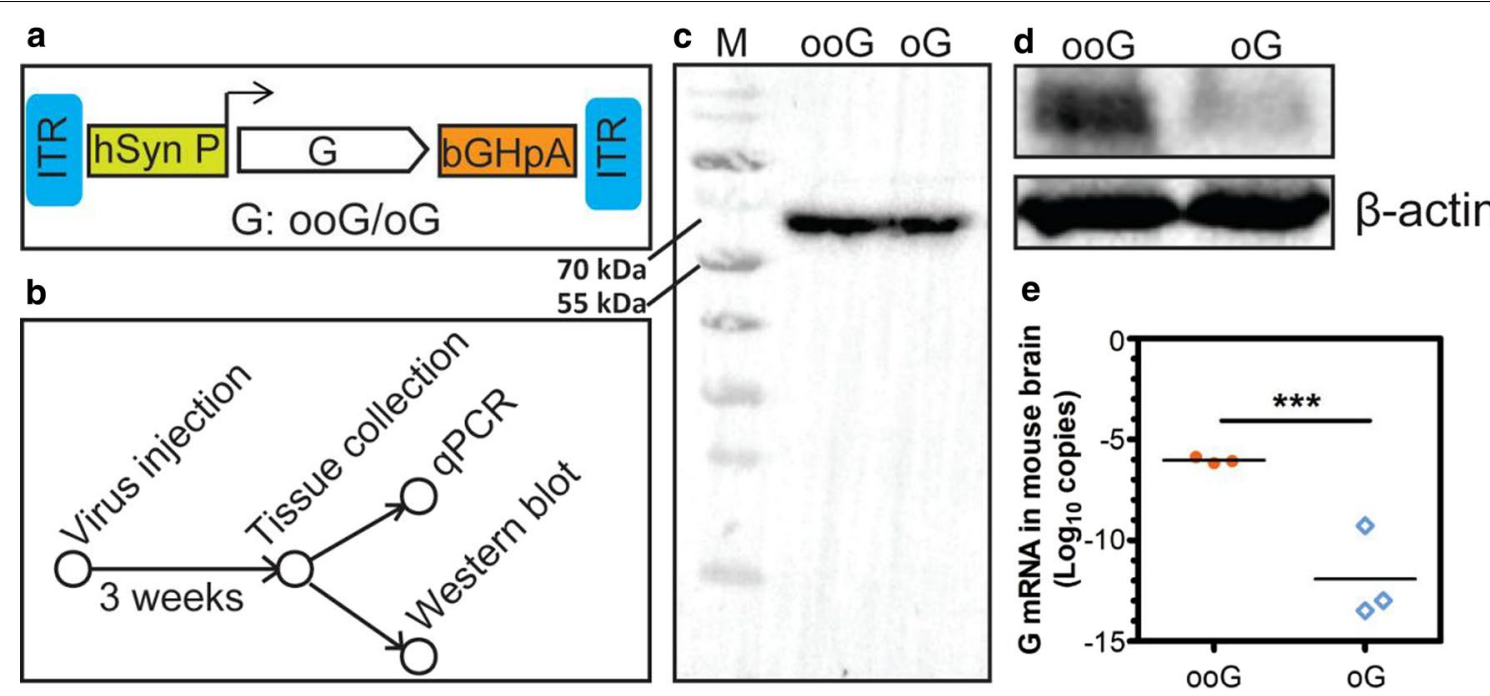

Fig. 3 The characteristics of the ooG and oG in mouse. a Design of sCAAV helper viruses express various RV G genes (ooG and oG). b The workflow of viruses labeling and sample treating. $\mathbf{c}$ The specificity of the antibody against the ooG and the oG was determined by western blot. $\mathbf{d}$ The ooG and $\mathrm{G}$ protein expression in mice brains were measured by Western blotting using antibody against the ooG. $\mathbf{e}$ The mRNA copies of the ooG and oG in mice brains were examined using quantitative RT-PCR. The t-test was conducted to compare the difference of mRNA copies of the ooG and oG. "****"Represents a statistically significant difference. The $p$-value of the ooG versus OG is 0.0004 . The data is from the three independent experiments

cells (EGFP and mRuby3 positive). In PVA region, the convergence index is the $2.547 \pm 0.05,1.132 \pm 0.05$ and $0.041 \pm 0.01$ for ooG, oG and B19G, respectively (Fig. 4b). In MS region, the convergence index is the $1.017 \pm 0.04,0.320 \pm 0.02$ and $0.061 \pm 0.01$ for the ooG, oG and B19G, respectively (Fig. 4c). Comparison with the data from the ooG, oG and B19G, we found that the convergence index of ooG is 2.3-fold and 62.1-fold higher than the oG and B19G, and the convergence index of oG is 27.6-fold higher than the B19G in PVA region which is similar with previous report [15]. The convergence index of the ooG is 3.2-fold and 16.7-fold higher than the oG and B19G, and the convergence index of the oG is 5.2-fold higher than the B19G in MS region. In addition, the convergence index is the $0.113 \pm 0.006$ and $0.009 \pm 0.002$ for the ooG and oG in lateral preoptic area (LPO), and the convergence index of the ooG is 12.6-fold higher than the oG (Fig. 5).

In addition, we compared the effects of the ooG and oG on labeling specificity and cellular toxicity. Firstly, the whole-brain connections to the vHPC were analyzed to investigate the labeling specificity, which revealed that the same brain regions were labeled in the ooG and oG groups (Additional file 1: Fig. S3a). These results indicated that the labeling specificity of the ooG is consistent with the oG. Secondly, the cellular toxicity of the ooG and the oG were determined by detecting the caspase- 3 and cell morphology. These results showed that the signals of caspase- 3 is not obvious in the ooG and oG groups (Additional file 1: Fig. S3b), and the cell morphologies in ooG and oG groups are similar (Additional file 1: Fig. S3c).

\section{Discussion}

To label the complete pre-synaptic and post-synaptic partners of a specific neuron is the ultimate goal for the neural circuit tracers. RV as the most important and popular tool has been used widely. However, the current systems can only label a fraction of presynaptic neurons [7]. Previous studies showed that the amount of the $G$ protein is one of the key steps for adjusting the trans-synaptic efficiency [15]. Therefore, many efforts have been taken for elevating the expression level of $\mathrm{G}$ protein. The direct method is using the stronger promoter to enhance the protein amount. Various promoters have different ability of improving gene expression in various cell types [22, 23]. Miyamichi et al. select CAG as a stronger promoter to drive $G$ expression [14]. Screening different $G$ proteins from various rabies virus strains is another method for improving RV trans-synaptic tracing efficiency. Kim et al. compared various G (N2C strain and Pasteur strain) and engineered a chimeric G (oG) using codon-optimized strategy [15]. In addition, providing more copies of the $\mathrm{G}$ gene might be a simple method for increasing gene expression. While the package capacity of AAV is limited. Therefore, screening and optimizing $G$ gene is an appropriate method for increasing the gene expression to improve RV trans-synaptic tracing efficiency.

During the long-term evolution, virus has adapted its host's environment for reproducing. All mammals are 


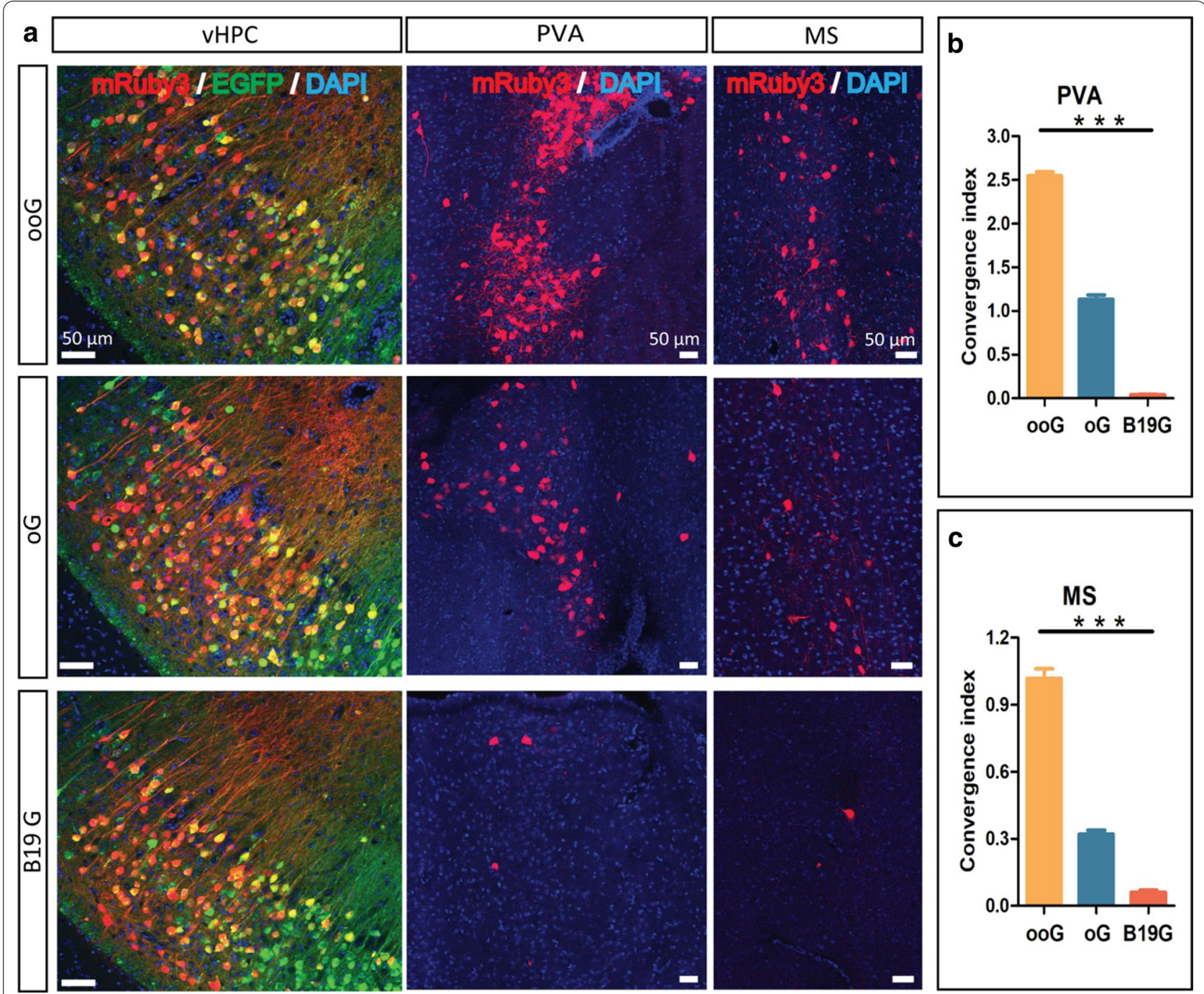

Fig. 4 Trans-monosynaptic tracing efficiency of RV with the help of various glycoproteins (ooG, oG and B19G). a Coronal sections were collected and imaged. Representative images of EnvA-RV-mRuby3 (EnvA-RV844) retrogradely trans-monosynaptic tracing from the vHPC to PVA with the help of two AAV helpers. b and c Convergence indices for long-distance projection inputs in PVA (b) and MS (c) using the ooG, OG, and B19G, respectively. The one-way ANOVA was conducted to compare the difference of RV tracing efficiency using different G genes (GraphPad Prism 5 software). Three mice for each $G$ gene. "***" Represents a statistically significant difference. Definitely, the p-values of the ooG versus oG, ooG versus B19G and OG versus B19G are less than 0.0001 , respectively. Error bars indicate the standard error of the mean from the three independent experiments

susceptible to rabies virus infection, only a few species are its reservoirs. Therefore, virus gene codons and gene codon pairs are adaptive to its reservoirs. Canine is one of RV reservoirs, while mouse is not. Therefore, the replicating and packaging efficiency of RV is not optimal in mouse. Mouse is the most important animal model for neuroscience research. Reshuffling virus genes according to the mouse genetic context might provide the proper condition for virus life cycle. CPB is a stable characteristic of a species. Based on the CPB strategy, attenuated viruses have been developed using under represented codon pair to deoptimize gene in RNA viruses and DNA viruses [17-19, 24-26]. In these cases, deoptimized gene has rare codon pairs and more $\mathrm{CpG}$ and $\mathrm{UpA}$, which might cause the lower gene expression. Several mechanism of virus attenuation were appeared, (i) under represented codon pair results in decreasing efficiency of translation, such as translation elongation rate, translation initiation complex dissociation, and protein processing [17, 19, 24, 26, 27], (ii) more CpG and UpA result in inducing an innate immune response and reducing mRNA stability [28-31]. However, the exact mechanism 


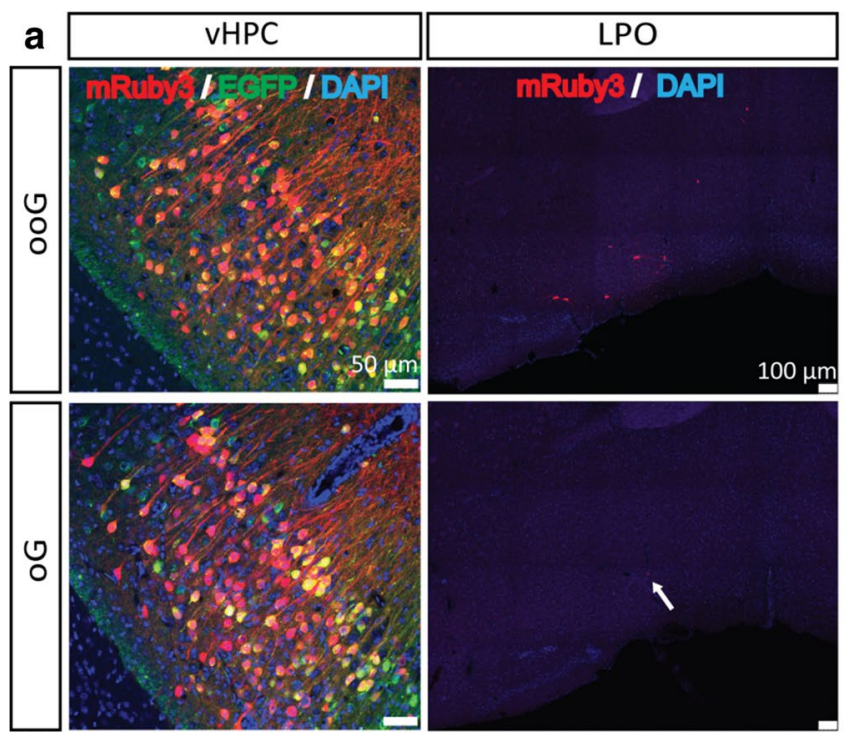

\section{b}

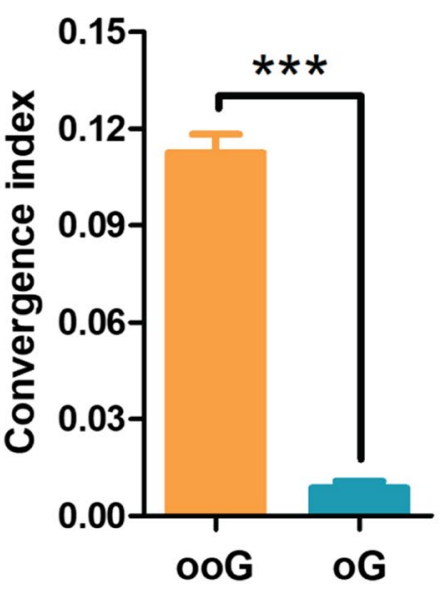

Fig. 5 Trans-monosynaptic efficiency of RV with the help of the ooG and oG. a Coronal sections were collected and imaged. Representative images of Enva-RV844 retrogradely trans-monosynaptic tracing from the VHPC to LPO with the help of two AAV helpers. b Convergence indices for projection inputs in the LPO using OOG and oG. The t-test was conducted to compare the difference of RV tracing efficiency using different $\mathrm{G}$ genes. Three mice for each G gene. ${ }^{\prime * * * "}$ Represents a statistically significant difference. The $p$-value of ooG versus oG is less than 0.0001 . Error bars indicate the standard error of the mean from the three independent experiments

of virus attenuation is still disputed and needs to be determined.

Unlike these previous studies, we provided a hypothesis that optimize gene using overrepresented codon pair to improve protein expression according to the mouse genome characteristic. Indeed, the ooG protein expression amount is higher than the oG (Fig. 3d, e). Therefore, ooG can significantly increase the RV transsynaptic efficiency (Fig. $4 \mathrm{a}-\mathrm{c}$ ). The characteristics of the oG and ooG in mouse are that the CPBS is -0.0738 and 0.3114 , the number of $\mathrm{CpG}$ is 73 and 38 , and the number of UpA is 45 and 34, respectively (Fig. 2c). Based on the results and possible mechanism for virus attenuated, we proposed that the over represented codon pair ooG provides a suitable situation for elevating translation efficiency, reducing an innate immune response and helping mRNA stability. However, the real mechanism should be determined in future. Collectively, whatever the mechanism for $\mathrm{CPB}$, it has become increasingly clear that $\mathrm{CPB}$ has a profound impact on protein expression.

In the present study, using RV as a model target and trans-synaptic tracing efficiency as a readout, we verified our hypothesis that nucleic acid sequence optimization based on CPB analysis is a convenient and efficient strategy to improve protein expression level. The ooG will be a useful tool for mapping neural circuits. Importantly, the approach reported here might provide a convenient, efficient and universal strategy to improve protein expression for various application scenarios such as other tracers, cell engineering, vaccine and oncolytic virus designs.

\section{Materials and methods}

\section{Animals}

All procedures were approved by the Animal Care and Use Committees at the Shenzhen Institute of Advanced Technology or Innovation Academy for Precision Measurement Science and Technology, Chinese Academy of Sciences. Eight-week-old male C57BL/6 mice were used for testing the protein expression and mRNA level. Eightweek-old Thy1-Cre mice were used for analyzing the retrograde trans-synaptic efficiency of rabies virus with the trans-complementary with various version $G$ proteins, which expresses Cre in the nervous system of transgenic mice.

\section{Rearrangement of the oG}

Firstly, the codon pair bias of mouse, canine and human were calculated using the previous method [17] based on the gene CDS annotation of each species from the Ensembl database release 98, which were based on the genome assembly GRCm38, CanFam3.1 and GRCh38. p13, respectively. Secondly, the oG gene was optimized based on the mouse CPS according to the general rule of maintaining the mean free energy of the RNA folding 
within a suitable range and to avoid dramatic changes in its secondary structure.

The algorithm to calculate CPS and CPBS for coding sequence was developed after the description of Kunec et al. [16] and Coleman et al. [17]. For a specific codon pair, the CPS is defined as the natural logarithm of the result of the observed codon pairs number divided by the expected number of codon pairs in all coding sequences of a species. The observed number is the actual number of the codon pair in the coding sequences. And the expected number of a codon pair is a theoretical value based on the proportion of amino acid and codon of the codon pair.

$$
C P S=\ln \left(\frac{O b s}{E x p}\right)
$$

where

$$
\operatorname{Exp}=\frac{N\left(\operatorname{codon}_{1}\right) \times N\left(\operatorname{codon}_{2}\right)}{N\left(a a_{1}\right) \times N\left(a a_{2}\right)} \times N\left(a a_{1} a a_{2}\right)
$$

The $N\left(\operatorname{codon}_{1}\right)$ and $N\left(\operatorname{codon}_{2}\right)$ denote the number of occurrences of two codons in a codon pair, respectively. The $N\left(a a_{1}\right)$ and $N\left(a a_{2}\right)$ are the number of corresponding amino acids. And $N\left(a a_{1} a a_{2}\right)$ is the number of the amino acid pair for the codon pair above. A positive CPS suggested the codon pair is over represented in the species, whereas a negative value is under represented.

Then, the CPBS for a coding sequence is the result of the arithmetic mean of all codon pair CPS values.

$$
C P B S=\sum_{i=n}^{n} \frac{C P S_{i}}{n-1}
$$

\section{Preparation AAV and RV}

scAAV-hsynP-ooG-bGHpA, scAAV-hsynP-oGbGHpA, and scAAV-hsynP-B19G-bGHpA were constructed by inserting the expression cassette into the self-complementary AAV (scAAV) core vector [20]. ssAAV-EF1 $\alpha$-ooG-WPRE-bGHpA and ssAAV-EF1 $\alpha$ DIO-EGFP-F2A-TVA-WPRE-bGHpA were constructed by inserting the expression cassette into the single-strand AAV2 core vector (addgene 20298). All plasmids were confirmed by sequencing. Then these recombinant AAVs were packaged with the AAV9 capsid, respectively.

Previous report shows that the mRuby3 is a new version red fluorescent protein with high lighter and stabilization [32]. Therefore, the mRuby3 gene was selected and inserted into pSADdeltaG-F3 vector [33]. Then the plasmid was confirmed by sequencing. The EnvA-RVmRuby3 (EnvA-RV844) was prepared using the previous method [2].

\section{Stereotaxic microinjection in mice brains}

Briefly, mice were anesthetized with chloral hydrate before injection. scAAV-hsynP-ooG-bGHpA $\left(6.9 \times 10^{11}\right.$ $\mathrm{vg} / \mathrm{ml})$, scAAV-hsynP-oG-bGHpA $\left(1.1 \times 10^{12} \mathrm{vg} / \mathrm{ml}\right)$ and scAAV-hsynP-B19G-bGHpA $\left(2 \times 10^{12} \mathrm{vg} / \mathrm{ml}\right)$ were respectively mixed $(1: 1$, total volume $80 \mathrm{nl})$ with ssAAVEF1 $\alpha$-DIO-EGFP-F2A-TVA-WPRE-bGHpA $\quad\left(6.2 \times 10^{11}\right.$ $\mathrm{vg} / \mathrm{ml}$ ) and co-injected into the vHPC region of mice. Then, the EnvA-RV-mRuby3 (EnvA-RV844, $2 \times 10^{8} \mathrm{FFU} /$ $\mathrm{ml}$ ) was injected into the same region after three weeks. During the process of virus injection, syringes were left in place for $10 \mathrm{~min}$ following injections to minimize diffusion. All surgical mice were back to the animal facility for extra seven days for fluorescent protein expression and RV trans-synaptic spreading.

\section{Brain section and immunohistochemistry}

Brain sections were collected referred in previous studies [13]. Briefly, mice were anaesthetized and transcardially perfused with $0.9 \%$ saline followed by $4 \%$ paraformaldehyde solution. The brains were removed and post-fixed over-night in $4 \%$ paraformaldehyde before being sectioned into $50 \mu \mathrm{m}$ slices, and slices were stained with DAPI. For staining the caspase-3, fixed slices were immunostained with the caspase- 3 antibody (1:500, Cell Signaling, \#9661) and amplified with the goat anti-rabbit secondary antibody (1:500, Jackson, \#611-605-215), and slices were stained with DAPI. Slices were imaged using the Leica TCS SP8 confocal microscope or the Olympus VS 120 slide scanning system.

\section{Preparation of antibody against the ooG}

$\mathrm{BALB} / \mathrm{c}$ mice were immunized directly by intramuscular injection of $200 \mu \mathrm{l}$ of AAV mixture with phosphate buffered saline, which contains $20 \mu \mathrm{l}$ of ssAAV-EF1 $\alpha$ ooG-WPRE-bGHpA $\left(1.4 \times 10^{12} \mathrm{vg} / \mathrm{ml}\right)$. Two weeks later, the second immunization was performed by intramuscular injection of the same amount AAV and the sera were collected after two weeks. Then, the specificity of the antibody was confirmed by using western blot to detect the expression of the ooG and the oG in the $293 \mathrm{~T}$ cells, which were separately transfected with $2 \mu \mathrm{g}$ of plasmids containing the expression cassettes of the ooG (pAAV-Ef1a-ooG-WPRE-BGHpA) and the oG (pAAV-Ef1a-oG-WPRE-BGHpA).

\section{Western blot}

$150 \mathrm{nl}$ of scAAV-hsynP-ooG-bGHpA $\left(8.7 \times 10^{11} \mathrm{vg} /\right.$ $\mathrm{ml})$ and scAAV-hsynP-oG-bGHpA $\left(1.3 \times 10^{12} \mathrm{vg} / \mathrm{ml}\right)$ were injected into the $\mathrm{vHPC}$ region of $\mathrm{C} 57 \mathrm{BL} / 6$ mouse, respectively. After three weeks, the tissue was collected and treated, one part for RNA extraction, and another 
for western blot. The tissue lysate or $293 \mathrm{~T}$ cells were separately analyzed on a $12 \%$ SDS-PAGE, and then electro-transferred to PVDF Immobilon-P membranes (Millipore), blocked with 5\% skim milk in TBST, and then treated with antibody against ooG at 1:500 dilution for $1 \mathrm{~h}$ at room temperature. After washing 3 times in TBST, the secondary anti-mouse antibody conjugated to Horseradish peroxidase at 1:5000 dilution was applied to the blots for $1 \mathrm{~h}$ at room temperature. To quantify the expression amount of GAPDH, the protein was analyzed by using fisrt antibody (1:2000) and second antibody (1:5000). Signal was detected with ECL western blotting reagent.

\section{Quantitative real-time PCR}

Total RNA was extracted from tissue using TRIzol Reagent. DNA was digested using DNaseI for $30 \mathrm{~min}$ at $37^{\circ} \mathrm{C} .1 \mu \mathrm{g}$ of RNA was reverse transcribed into cDNA using random hexamers. Specific primers (for ooG, forward primer, GGCCTACAACTGGAAGATGGC, for oG, forward primer, AGCTTACAACTGGAAGATGGC, and they have the same reverse primer, TAGAAGACACCG CTACTCCT, for GAPDH, forward primer, GGTGAA GGTCGGTGTGAACG, reverse primer, CTCGCTCCT GGAAGATGGTG) were used to quantify by qPCR using the SYBR Green Master Mix on the real-time PCR detection system (Bio-Rad). The copy numbers were determined based on standard curve generated for each gene using known concentration plasmids pSyn-ooG, pSynoG, and pcDNA-GAPDH.

\section{Supplementary Information}

The online version contains supplementary material available at https://doi. org/10.1186/s13041-021-00821-7.

\footnotetext{
Additional file 1: Fig. S1. Distribution of codon pair bias scores in human, canine and mouse. CPBSs were calculated using the previous described method [17], Each salmon red circle represents a CPBS of a single human (a), canine (b), and mouse (c) gene plotted against its gene length, respectively. The average CPBSs of the human, canine and mouse are 0.0703 , 0.0704 , and 0.0651 , respectively. Fig. S2. The sequence of the ooG. The ooG sequence was produced based on the oG [15] by using a previous described method [17]. > ooG sequence ATGGTTCCTCAAGCCCTTCTCTTT GTTCCTCTTCTTGTCTTCCCGCTCTGCTTTGGGAAGTTCCCCATCTACACCATT CCTGACAAGCTAGGGCCCTGGAGCCCCATTGACATCCACCACCTCAGCTGC CCCAACAACTTGGTTGTAGAAGATGAAGGCTGCACCAACCTCAGTGGCTTC TCCTACATGGAGCTAAAAGTGGGCTACATCTCGGCCATCAAGATGAATGGC TTCACCTGCACTGGAGTTGTCACTGAAGCAGAGACCTACACCAACTTTGTT GGCTATGTCACCACCACCTTCAAAAGAAAACACTTCCGGCCCACTCCAGAT GCCTGCCGCGCGGCCTACAACTGGAAGATGGCGGGGGACCCCCGCTATGAA GAGAGCCTGCACAACCCCTACCCAGACTACCACTGGCTGAGGACTGTGAAG ACCACCAAAGAAAGTTTGGTCATCATCAGCCCCAGTGTAGCTGACTTGGAC CCCTATGACCGTTCTCTACACAGCCCTGTATTTCCTGGTGGGAACTGCAGTGGT GTGGCTGTCAGCAGCACCTACTGCAGCACCAACCATGACTACACCATCTGG ATGCCGGAGAACCCCCGGCTAGGGATGTCCTGTGACATCTTCACCAACAGC CGAGGGAAAAGAGCCAGCAAAGGTTCTGAGACCTGTGGCTTTGTAGATGAG CGTGGCCTCTACAAGAGTTTAAAAGGTGCCTGCAAATTAAAACTCTGTGGT GTTCTTGGTCTTCGGCTCATGGATGGCACCTGGGTGGCCATGCAGACCAGC AATGAGACCAAGTGGTGCCCGCCGGGCCAGCTTGTCAACCTCCATGACTTC
}

CGAAGTGATGAAATAGAACATCTTGTTGTAGAAGAACTTGTCAAGAAAAGA GAAGAATGTTTAGATGCCCTGGAGAGCATCATGACTACCAAGAGTGTCTCC TTCCGTCGCCTCAGCCACCTCAGGAAACTTGTTCCTGGCTTTGGGAAAGCC TACACCATCTTCAACAAGACGCTCATGGAAGCAGATGCCCACTACAAATCT GTCCGCACGTGGAATGAGATCATTCCTTCCAAAGGCTGCCTCCGAGTTGGT GGCCGCTGCCACCCACATGTCAATGGTGTCTTCTTCAATGGCATCATTCTTGGG CCAGATGGAAATGTCCTCATTCCAGAGATGCAGAGCAGCCTGCTGCAGCAG CACATGGAACTTCTTGTCAGCAGTGTCATCCCGCTCATGCACCCGCTGGCA GACCCCAGCACTGTCTTCAAGAATGGAGATGAAGCAGAAGATTTTGTAGAA GTTCATCTTCCTGATGTTCATGAAAGAATTTCTGGTGTGGACTTGGGTCTTCCC AACTGGGGAAAATATGTTCTTCTTTCTGCTGGGGCGCTCACGGCGCTCATG TTAATAATATTCCTCATGACCTGCTGCAGAAGAGTCAACCGCTCGGAGCCCACC CAGCACAACCTTCGTGGCACGGGCCGAGAAGTTTCTGTCACGCCGCAGAGT GGGAAGATCATCTCCTCCTGGGAGAGCCACAAGTCAGGAGGAGAGACGCGC CTGTAA. Fig. S3. The effects of the ooG on labeling specificity and cellular toxicity. (a) The percentage of input neurons from each site to total quantified inputs in the ooG and oG groups. (b) The signals of the caspase-3 was determined by immunohistochemistry using antibody against the caspase-3 in the $\mathrm{OOG}_{\mathrm{G}}$ and $\mathrm{OG}$ groups. (b) The fine structure of the neuron is apparent in the ooG and oG groups. These images are the representatives from three mice.

\section{Acknowledgements}

We are grateful to Dr. Xiao Xiao (University of North Carolina at Chapel Hill) for his kind gift of pdsAAV2-CBAp-EGFP plasmids, Dr. Robert Konrad Naumann for the proofreading.

\section{Authors' contributions}

FJ and FQX conceived of the project; FJ designed the experiments and generated the figures; FJ, LL, HZL, PL, XWS, YW, and CL preformed experiments; FJ and FQX wrote the manuscript. All authors read and approved the final manuscript.

\section{Funding}

This work was supported by the Key-Area Research and Development Program of Guangdong Province (2018B030331001), the National Natural Science Foundation of China (32071038, 31830035, 91732304, 91632303, 81661148053, and 31771156), the GuangDong Basic and Applied Basic Research Foundation (2021A1515011235), Shenzhen Key Laboratory of Viral Vectors for Biomedicine (ZDSYS20200811142401005), the SIAT Innovation Program for Excellent Young Researchers (E1G023), the National Basic Research Program (973 Program) of China (2015CB755600), the Strategic Priority Research Program (B) (XDB32030200).

Availability of data and materials

The data that used in this study are available from the corresponding author upon reasonable request.

\section{Declarations}

\section{Ethics approval and consent to participate}

All procedures were approved by the Animal Care and Use Committees at the Shenzhen Institute of Advanced Technology or Innovation Academy for Precision Measurement Science and Technology, Chinese Academy of Sciences.

\section{Consent for publication}

Not applicable.

\section{Competing interests}

The authors declare no conflict of interest.

\section{Author details}

${ }^{1}$ Guangdong Provincial Key Laboratory of Brain Connectome and Behavior, CAS Key Laboratory of Brain Connectome and Manipulation, The Brain Cognition and Brain Disease Institute (BCBDI), Translational Research Center for the Nervous System (TRCNS), Shenzhen Institute of Advanced Technology, Chinese Academy of Sciences, Shenzhen 518055, China. ${ }^{2}$ Shenzhen-Hong 
Kong Institute of Brain Science-Shenzhen Fundamental Research Institutions, Shenzhen 518055, China. ${ }^{3}$ NMPA Key Laboratory for Research and Evaluation of Viral Vector Technology in Cell and Gene Therapy Medicinal Products, Key Laboratory of Quality Control Technology for Virus-Based Therapeutics, Guangdong Provincial Medical Products Administration, Shenzhen Key Laboratory of Viral Vectors for Biomedicine, Shenzhen Institute of Advanced Technology, Chinese Academy of Sciences, Shenzhen 518055, China. ${ }^{4}$ State Key Laboratory of Magnetic Resonance and Atomic and Molecular Physics, Key Laboratory of Magnetic Resonance in Biological Systems, "Wuhan Center for Magnetic Resonance, Innovation Academy for Precision Measurement Science and Technology, Chinese Academy of Sciences, Wuhan 430071, China. ${ }^{5}$ Wuhan National Laboratory for Optoelectronics, Huazhong University of Science and Technology, Wuhan 430074, China. ${ }^{6}$ University of Chinese Academy of Sciences, Beijing 100049, China. 'Wuhan Institute of Virology, Chinese Academy of Sciences, Wuhan 430071, China. ${ }^{8}$ Center for Excellence in Brain Science and Intelligence Technology, Chinese Academy of Sciences, Shanghai 200031, China. ${ }^{9}$ Division of Molecular and Cellular Therapy, Department of Pediatrics, College of Medicine, University of Florida, Gainesville, FL, USA.

Received: 15 March 2021 Accepted: 4 July 2021

Published online: 08 July 2021

\section{References}

1. Nassi JJ, et al. Neuroanatomy goes viral! Front Neuroanat. 2015;9:80.

2. Wickersham IR, et al. Monosynaptic restriction of transsynaptic tracing from single, genetically targeted neurons. Neuron. 2007:53(5):639-47.

3. Wickersham $I R$, et al. Retrograde neuronal tracing with a deletion-mutant rabies virus. Nat Methods. 2007;4(1):47-9.

4. Reardon TR, et al. Rabies virus CVS-N2C(DeltaG) strain enhances retrograde synaptic transfer and neuronal viability. Neuron. 2016;89(4):711-24.

5. Chatterjee $\mathrm{S}$, et al. Nontoxic, double-deletion-mutant rabies viral vectors for retrograde targeting of projection neurons. Nat Neurosci. 2018;21(4):638-46.

6. Ciabatti $E$, et al. Life-long genetic and functional access to neural circuits using self-inactivating rabies virus. Cell. 2017;170(2):382-392 e14.

7. Callaway EM, Luo L. Monosynaptic circuit tracing with glycoproteindeleted rabies viruses. J Neurosci. 2015;35(24):8979-85.

8. Hong $\mathrm{S}$, et al. Functional analysis of various promoters in lentiviral vectors at different stages of in vitro differentiation of mouse embryonic stem cells. Mol Ther. 2007;15(9):1630-9.

9. Schlabach MR, et al. Synthetic design of strong promoters. Proc Natl Acad Sci U S A. 2010;107(6):2538-43.

10. Francis DM, Page R. Strategies to optimize protein expression in E. coli. Curr Protoc Protein Sci. 2010;24:1-29 (chapter 5, unit 5).

11. Zhou Z, et al. Codon usage is an important determinant of gene expression levels largely through its effects on transcription. Proc Natl Acad Sci U S A. 2016;113(41):E6117-25.

12. Srividya D, Shyam Mohan AH, Rao SN. Expression and purification of codon-optimized cre recombinase in E. coli. Protein Expr Purif. 2020;167:105546

13. Jia F, et al. Optimization of the fluorescent protein expression level based on pseudorabies virus bartha strain for neural circuit tracing. Front Neuroanat. 2019;13:63.

14. Miyamichi K, et al. Dissecting local circuits: parvalbumin interneurons underlie broad feedback control of olfactory bulb output. Neuron. 2013;80(5):1232-45.
15. Kim EJ, et al. Improved monosynaptic neural circuit tracing using engineered rabies virus glycoproteins. Cell Rep. 2016;15(4):692-9.

16. Kunec D, Osterrieder N. Codon pair bias is a direct consequence of dinucleotide bias. Cell Rep. 2016;14(1):55-67.

17. Coleman JR, et al. Virus attenuation by genome-scale changes in codon pair bias. Science. 2008;320(5884):1784-7.

18. Le Nouen C, et al. Attenuation of human respiratory syncytial virus by genome-scale codon-pair deoptimization. Proc Natl Acad Sci U S A. 2014;111(36):13169-74.

19. Shen $\mathrm{SH}$, et al. Large-scale recoding of an arbovirus genome to rebalance its insect versus mammalian preference. Proc Natl Acad Sci U S A. 2015;112(15):4749-54.

20. Ling $C$, et al. Selective in vivo targeting of human liver tumors by optimized AAV3 vectors in a murine xenograft model. Hum Gene Therapy. 2014;25(12):1023-34.

21. Paxinos G, Franklin KBJ. The mouse brain in stereotaxic coordinates. 2nd ed. San Diego, Calif. London: Academic. 2001; xxv, 264 p

22. Qin JY, et al. Systematic comparison of constitutive promoters and the doxycycline-inducible promoter. PLoS ONE. 2010;5(5): e10611.

23. Norrman K, et al. Quantitative comparison of constitutive promoters in human ES cells. PLoS ONE. 2010;5(8): e12413.

24. Wang $B$, et al. Recoding of the vesicular stomatitis virus $L$ gene by computer-aided design provides a live, attenuated vaccine candidate. MBio. 2015. https://doi.org/10.1128/mBio.00237-15.

25. Gao L, et al. HP-PRRSV is attenuated by de-optimization of codon pair bias in its RNA-dependent RNA polymerase nsp9 gene. Virology. 2015:485:135-44

26. Eschke K, et al. Attenuation of a very virulent Marek's disease herpesvirus (MDV) by codon pair bias deoptimization. PLoS Pathog. 2018;14(1): e1006857.

27. Meng J, et al. Refining the balance of attenuation and immunogenicity of respiratory syncytial virus by targeted codon deoptimization of virulence genes. MBio. 2014;5(5): e01704-14.

28. Gaunt $\mathrm{E}$, et al. Elevation of $\mathrm{CpG}$ frequencies in influenza A genome attenuates pathogenicity but enhances host response to infection. Elife. 2016;5: e12735.

29. Burns CC, et al. Genetic inactivation of poliovirus infectivity by increasing the frequencies of CpG and UpA dinucleotides within and across synonymous capsid region codons. J Virol. 2009;83(19):9957-69.

30. Al-Saif M, Khabar KS. UU/UA dinucleotide frequency reduction in coding regions results in increased mRNA stability and protein expression. Mol Ther. 2012;20(5):954-9.

31. Tulloch $F$, et al. RNA virus attenuation by codon pair deoptimisation is an artefact of increases in CpG/UpA dinucleotide frequencies. Elife. 2014;3: e04531.

32. Bajar BT, et al. Improving brightness and photostability of green and red fluorescent proteins for live cell imaging and FRET reporting. Sci Rep. 2016;6:20889.

33. Osakada F, et al. New rabies virus variants for monitoring and manipulating activity and gene expression in defined neural circuits. Neuron. 2011;71(4):617-31.

\section{Publisher's Note}

Springer Nature remains neutral with regard to jurisdictional claims in published maps and institutional affiliations. 